\title{
L'Herbe face à la critique
}

Gérard Roubichou

\section{(2) OpenEdition}

Journals

Édition électronique

URL : https://journals.openedition.org/ccs/598

DOI : $10.4000 /$ ccs.598

ISSN : 2558-782X

\section{Éditeur :}

Presses universitaires de Rennes, Association des lecteurs de Claude Simon

\section{Édition imprimée}

Date de publication : 30 novembre 2008

Pagination : 77-93

ISBN : 9782354120351

ISSN : 1774-9425

\section{Référence électronique}

Gérard Roubichou, «L'Herbe face à la critique », Cahiers Claude Simon [En ligne], 4 | 2008, mis en ligne le 21 septembre 2017, consulté le 25 avril 2022. URL : http://journals.openedition.org/ccs/598 ; DOI : https://doi.org/10.4000/ccs.598 


\section{L'Herbe face à la critique}

\section{Gérard ROUBICHOU*}

« [...] À partir de L'Herbe, mes livres sont tous à base de vécu, expression que je préfère à "autobiographie" ».

\section{En guise d'introduction}

Qu'on me permette, en ma qualité d'ancien, quelques remarques personnelles au début de cet exposé. Tout d'abord, je me réjouis, à l'occasion de ce séminaire ${ }^{1}$, de « retrouver » L'Herbe qui fut pour moi à l'origine d'une longue amitié de plus de trente ans avec Claude Simon. C'est, en effet, au printemps 1970, que nous nous rencontrâmes pour la première fois à New York. J'étais alors un jeune universitaire français aux États-Unis. J'avais décidé de consacrer ma thèse de doctorat à Claude Simon qui était aussi le sujet d'un de mes cours. Mais je fus amené à la présenter... en Suisse (car on ne pouvait, en ces temps-là, envisager en France une thèse sur un auteur vivant). Au départ, mon sujet était plutôt ambitieux : « Le cycle des Reixach : L'Herbe, La Route des Flandres, Le Palace et Histoire ». Diverses considérations universitaires (matière trop abondante et, par conséquent, risque de délais trop longs) devaient, dans les deux années suivantes, m'imposer de me limiter à un seul roman. Je choisis donc de me consacrer à L'Herbe. Ce «roman», dont la lecture avait été pour

\footnotetext{
* Paris.

1 Le séminaire de l'Association des Lecteurs de Claude Simon consacré à L'Herbe, d'où sont issus les trois articles de ce dossier, s'est tenu le 10 juin 2006 dans les locaux de l'École Normale Supérieure de la rue d'Ulm (note de la rédaction).
} 
moi une expérience aussi importante que celle du Vent, me semblait constituer alors une première étape dans le développement de la production romanesque de Claude Simon qui, à ce moment-là, avait déjà publié, rappelons-le, La Bataille de Pharsale (1969) et préparait Les Corps conducteurs (1971).

Tout au long de la période de recherches, de composition et de rédaction de ce travail universitaire de 1971 à 1973 - date de la soutenance - et bien longtemps encore après sa publication " commerciale », j'eus le privilège d'entretenir, malgré l'éloignement, un dialogue amical et soutenu avec Claude qui fut, en fait, mon premier lecteur et « critique " non universitaire. Il partagea même, à travers une correspondance suivie, mes frustrations dues à des circonstances contraires et indépendantes de ma volonté qui eurent pour effet de retarder à 1976 la publication «commerciale» de ma Lecture de L'Herbe'.

Comment pourrais-je ne pas évoquer ce contexte particulier dans cette rencontre d'aujourd'hui ? Et comment, du même coup, ne pas rendre hommage à l'écrivain Claude Simon disparu il y a un peu moins d'un an ? C'est ce qui m'a décidé à me manifester en participant à ce débat. Le choix de L'Herbe. Il y avait bien longtemps, je l'avoue, que je n'avais pas relu ce roman. Et l'ayant relu, je continue à penser qu'il occupe bien une place particulière (à part ?) entre $L e$ Vent (1957) et Le Tramway (2001), c'est-à-dire dans ce cheminement des œuvres qui n'a fait que confirmer Claude Simon comme un " énorme » écrivain contemporain français au point de lui valoir en toute légitimité d'entrer dans la Pléiade au printemps 2006.

\section{L'édition de « La Pléiade » et L'Herbe}

La coïncidence de dates entre cette rencontre d'aujourd'hui autour de L'Herbe et la publication d'Euvres de Claude Simon dans la Pléiade n'a pas été nécessairement voulue. Mais précisément puisque L'Herbe ne figure pas dans la sélection des œuvres de Claude Simon retenues dans cette édition qui se présente comme une intro-

\footnotetext{
${ }^{1}$ Gérard Roubichou, Lecture de L'Herbe de Claude Simon, Editions L'Âge d'Homme, « Lettera », Lausanne, 1976, 337 p.
} 
duction à l'univers du romancier, on ne pouvait trouver une meilleure opportunité d'en parler! Saluons ici la sagesse de l'Association.

Je partirai d'une déclaration récente d'Alastair Duncan pour tenter de situer mon propos dans cette communication sur L'Herbe

Quand on m'a contacté, Claude Simon avait déjà fait son choix. Mis à part les premiers romans, qu'il prisait peu, toutes les époques de son travail sont présentes : le cycle des romans de la mémoire restituante, y compris ce chef-d'œuvre qu'est La Route des Flandres ; l'important roman de transition, La Bataille de Pharsale; la période dite formaliste représentée par Triptyque; et le roman à base de vécu, dont nous avons ce magnifique fleuron, Le Jardin des Plantes. Deux facteurs ont pu jouer dans le choix de Simon. D'abord, peut-être, le désir de mettre en avant des romans qu'on avait un peu négligés, Le Lent ou Le Palace. Alors que L'Acacia ou Le Tramway étaient déjà célèbres et célébrés. Et puis, parmi les romans que Simon n'a pas choisis, on trouve L'Acacia, Les Géorgiques, Histoire et L'Herbe. Tous ces romans traitent plus ou moins des sujets se rapportant à son histoire familiale. Or l'écrivain savait qu'une édition de la Pléiade s'intéresserait, entre autres, aux origines de l'œuvre ; il avait horreur de passer pour un chroniqueur de la vie familiale. Il se méfiait donc de tout ce qui pouvait tirer son œuvre dans ce sens. L'essentiel pour lui était le travail sur l'écriture. ${ }^{1}$

Les remarques que je vais faire ne visent aucunement à sousestimer les contraintes juridiques, voire commerciales (protection de certains " copyrights ») et les orientations éditoriales fixées pour cette publication à 1500 pages, même si je déplore que le seul écrivain français sans aucun doute le plus représentatif de la vitalité de notre langue et de notre littérature dans les cinquante dernières années et le dernier à avoir été consacré par le Prix Nobel de littérature n'ait pas eu « droit» à des œuvres... plus « complètes ». Je n'en-

1 Alastair Duncan : «La preuve que l'homme peut imposer un sens », Le Monde des Livres, 17 février 2006, p. 6. Voir aussi, «Dans la forêt des mots », interview d'Alastair Duncan, Le Matricule des Anges, n ${ }^{\circ}$ 72, avril-mai 2006, pp. 18-20, où sont reprises et précisées certaines phrases de ces déclarations. 
tends pas non plus critiquer les choix du romancier obligé dans ce contexte, à ce que je comprends, de "se s(él)ectionner»; et, enfin, mes observations ne sont aucunement une manière de déprécier le remarquable et impressionnant travail de recherche et $\mathbf{d}^{\prime}$ " édition» accompli par les deux co-auteurs du volume de La Pléiade, Alastair B. Duncan et Jean H. Duffy ${ }^{1}$.

Je retiendrai quand même de la version « officielle » que je crois lire à travers cette citation que la distribution artificielle et inévitable dans tout processus de sélection se révèle en l'occurrence réductrice autant par les catégories particulières qui auraient servi à départager les œuvres (romans de la "mémoire restituante»; roman de transition ; roman de la période formaliste ; roman à base de vécu) que par certaines des œuvres retenues qui auraient pu fort bien être remplacées avantageusement par d'autres ; en outre, elle s'appuie sur une référence générique - roman - considérée comme allant de soi.

Considérons, d'abord, les motifs d'exclusion de L'Herbe. Ils peuvent étonner : le premier est sa qualité d'œuvre traitant "plus ou moins des sujets se rapportant à [1]'histoire familiale [de l'auteur] ", statut qu'elle partagerait avec trois autres productions capitales de l'œuvre simonienne, L'Acacia, Les Géorgiques et Histoire! Comme si ces dernières et L'Herbe couraient le risque d'être prises pour des « chroniques» de la vie familiale! Ensuite, ces quatre romans étant des œuvres «à base de vécu $\|^{2}$ ne pourraient rendre compte du travail de l'écrivain et risqueraient de détourner de «l'essentiel», c'està-dire « le travail sur l'écriture ». Or, c'est bien pourtant ce qui, depuis L'Herbe, constitue une des données fondamentales de l'univers romanesque de Claude Simon. L'Herbe, en étant, selon moi, la première manifestation, ne ferait pas exception... Je dirais même que la séquence - Le Vent, L'Herbe, La Route des Flandres, le Palace - (romans de la "mémoire restituante» avant beaucoup d'autres) constituerait une excellente introduction à la découverte des fondements de l'uni-

Depuis que cette communication a été prononcée, l'édition d'un second volume d'œuvres a été décidée, dans lequel figurera L'Herbe. Cette édition sera établie par Alastair Duncan, assisté de David Zemmour.

Claude Simon ne disait-il pas, en 1989: «À partir de L'Herbe, mes livres sont tous à base de vécu, expression que je préfère à "autobiographie" » (L'Événement dujeudi, 31 août-6 septembre 1989) ? Cette constatation apparaît dans de nombreuses déclarations faites par l'auteur à la même époque. 
vers « romanesque » simonien par la mise en place progressive des données d'un ensemble marqué par un travail sur l'écriture destiné à se développer, s'amplifier, voire se réorienter par la suite.

Mon second point est nettement plus complexe. En effet, si l'on excepte certains textes non discursifs (théoriques, poétiques ou esthétiques), toutes les œuvres de "fiction" de Claude Simon - du Vent au Tramway - sont qualifiées (du moins sur leur couverture) de « romans ». Or il apparaît à l'évidence - si l'on me permet d'user de cette expression - que ce pavillon couvre différentes sorte de productions. De quel « roman » peut-on parler à propos de L'Herbe ou, pour être plus exact, de quel étape du « roman» simonien L'Herbe témoignerait-il ? Et que dire du "roman» simonien lui-même? Vaste programme que - je vous rassure tout de suite - je ne ferai qu'effleurer, mais qui me paraît ne pas devoir être éludé.

\section{Le statut de L'Herbe}

Ce qui m'a frappé, en retournant vers $L^{\prime} H e r b e$ pour me préparer à cette présentation, c'est, depuis sa publication, la singularité de son statut dans la production romanesque simonienne. Si, pour reprendre les termes d'Alastair Duncan, Le Vent et Le Palace sont des " romans qu'on avait un peu négligés ", ne pourrait-on pas le dire aussi bien de $L^{\prime} H_{e r b e}{ }^{1}$ ? Et que peut-on entendre par là ? J'ignore quels furent, en comparaison de ces deux romans, les tirages de L'Herbe, à supposer qu'ils représentent un critère déterminant pour évaluer l'ampleur de la réception d'un "roman un peu négligé $»^{2}$. Je crois me souvenir qu'il y a eu au moins deux rééditions (1962, 1971) jusqu'à celle de 1986 qui comportait une postface d'Alastair Duncan (avec un nouveau tirage en 2005). Une chose est toutefois sûre, c'est que, bien que, comme nous allons le voir, ce roman n'ait pas été ignoré, L'Herbe n'a pas non plus été sur « le chemin » qu'ont parcouru à partir d'un certain moment les études sur Claude Simon.

1 En 1973, Jérôme Lindon, dans une lettre personnelle qu'il m'adressait, désignait L'Herbe comme «l'une [des œuvres] les plus anciennes et l'une des moins connues de l'auteur".

2 Si l'on regarde dès 1972 les traductions de ces trois romans, ils sont à peu près sur le même plan : 2 traductions en anglais et entre 5 et 7 traductions dans d'autres langues. Voir Entretiens, Claude Simon, Editions Subervie, 1972, notamment pp. 182-184 "Éditions en langues étrangères ». 
En d'autres termes, il a été progressivement plus ou moins oblitéré, voire évacué. Et l'on peut au moins s'interroger sur le pourquoi de cette évolution qui tient (peut-être ?) à sa nature de « roman » à part dans l'ensemble de la production simonienne.

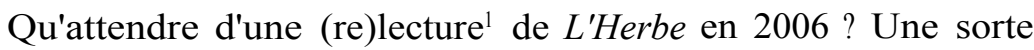
de réhabilitation ? Voilà un objectif à la fois bien présomptueux et vain. Alors plutôt une réflexion sur la « lisibilité » de L'Herbe comme œuvre simonienne, si l'on entend par là qu'en dépit de son caractère d'œuvre datée (1958), le roman reste en même temps une production « représentative de l'œuvre »-dont elle manifesterait certaines données capitales? Doit-on, même si ou peut-être parce qu'on est un lecteur averti, (re)lire L'Herbe en 2006 ? Et si oui, pourquoi et comment, d'une part, au regard d'une production simonienne qui s'est considérablement développée et, d'autre part, au regard des travaux critiques de plus en plus nombreux, voire pléthoriques, qui ont été consacrés à cette dernière mettant en perspective ses composantes successives à l'intérieur d'un « système global $»^{2}$ et ignorant assez souvent L'Herbe ? Pour ma part, je crois que la successivité des œuvres depuis 1957 reste une donnée non pas seulement «chronologique " (la suite des romans écrits par Claude Simon), mais aussi et surtout « organique » (leur « foisonnant et rigoureux désordre » (Hist., 273) en même temps qu'elle révèle un effet $\mathbf{d}^{\prime}$ '« emboîtement» tel que celui de «tables gigognes $»^{3}$ ), ce qui aurait pour conséquence collatérale d'écorner le projet même de sélection, sorte de "menu-dégustation », des œuvres de Claude Simon proposé par la Pléiade que je déplorais plus haut.

En d'autres termes, en quoi, sachant ce que l'on sait depuis sa pa-

1 Je donne à ce terme le sens $\mathbf{d}^{\prime}$ « expérience du lecteur» (empruntant un titre de Christine Genin), sans ignorer certaines définitions qui en ont été proposées sur un plan méthodologique par T. Todorov et que reprend Ralph Sarkonak au début de ses Carrefours du texte, Editions Paratexte, Toronto, 1986, notamment p. 7.

2 Ibid.

3 "Claude Simon, franc-tireur de la révolution romanesque », entretien de Claude Simon avec Thérèse de Saint-Phalle (Le Figaro Littéraire, 6 avril 1967) : "Mes livres sortent les uns des autres comme des tables gigognes. Je n'aurais pu écrire Histoire sans avoir écrit Le Palace, ni Le Palace sans La Route des Flandres. En général, c'est avec ce qui n'a pu être dit dans les livres précédents que je commence un nouveau roman. " Voir aussi cette phrase paradoxale, mais utile à notre propos : "L'Herbe, c'est la suite de La Route des Flandres. " (Hubert Juin, "Les secrets du romancier », Entretien avec Claude Simon, Les Lettres françaises, $\mathrm{n}^{\circ}$ 844, 6-12 octobre 1960). 
rution (il y a presque cinquante ans), L'Herbe peut-il être lu comme un roman simonien qui, bien qu'imparfait et encore marqué par les efforts, les « tâtonnements » de son auteur à se définir dans son écriture et son univers, appartient (et comment ?) à ce grand "Texte" simonien en sa qualité de produit d'une écriture, ce qui, chez Claude Simon, n'a cessé d'être « l'essentiel».

\section{Lectures de L'Herbe}

Depuis 1958, L'Herbe n'a pas été une des œuvres les plus ignorées de la critique principalement universitaire, mais ce roman a pris peu à peu - du moins à l'intention d'un plus large public - une orientation singulière. Il suffit déjà de comparer, par exemple, la quatrième de couverture de l'édition de 1962 à celle de l'édition de $1986^{1}$ : sans être radical, le changement est significatif de ce qu'on pourrait appeler, à tous les sens du terme, son « classement».

Mais avant de m'engager trop loin dans des conclusions issues d'une relecture plus personnelle que théoricienne, je me propose de tracer, dans un survol dont on excusera le caractère un peu schématique, un rapide panorama de la critique de L'Herbe que le remarquable travail bibliographique de Christine Genin a grandement facilité. En effet, l'évolution du statut de L'Herbe reflète et s'inscrit dans celle de la critique des œuvres de Claude Simon des quarante

Quatrième de couverture de l'édition de 1962: « Le sujet de L'Herbe, ce n'est pas cette jeune femme, Louise, ni ce qu'elle fait, ni ce qui lui arrive, ni même ce qu'elle ressent [...] et pourtant, c'est l'histoire elle-même (la façon quasi photoplasmique qu'elle a de se faire sans direction fixée d'avance, sans ordre apparent, sans répit) qui est l'unique objet du roman. ", " à travers chacune de ces touches, à l'intérieur de chacune de ces phrases en surgit une autre, et dans celles-ci une autre encore, indéfiniment, leur simultanéité constituant la texture d'une sorte de tissu organique, complexe et apparemment inerte ». Quatrième de couverture de l'édition de 1986 : «Le roman tourne autour d'une hésitation : Louise quittera-t-elle ou non son mari pour l'amant à laquelle elle exprime son trouble ? Cette question se double d'une autre : la vieille tante agonisante mourrat-elle pour de bon? Liant une jeune et une femme, ce roman situe le dilemme de son personnage central dans le cadre d'une famille déchirée... ». Ce dernier texte est une citation directe extraite du livre de Stuart Sykes publié en 1979 (voir note suivante). Toutefois, dans sa postface de 1986, Alastair B. Duncan écrit: «Le véritable drame de L'Herbe se joue dans une tension entre, d'une part, le pouvoir des mots, créateur et entraînant, et, d'autre part, une volonté de leur imposer continuité et cohérence." 
dernières années en fonction de trois orientations principales : la première, la plus importante en quantité de travaux et la plus longue, serait d'ordre " pédagogique »; la seconde correspondrait à une approche transversale plus "épistémologique»; la dernière à une approche que je désignerai, faute de mieux, d'approche transversale " thématique».

Dans la première, on relèverait pas loin d'une trentaine d'études (livres, chapitres de livres, articles de revues) d'ampleur et d'importance inégales qui ont été consacrées à L'Herbe à partir de sa parution en 1958 - soit dans un cadre monographique, soit à l'intérieur d'un même ouvrage comportant une série d'études successives des œuvres de Claude Simon à une certaine date, soit autour d'une thématique bien ciblée, soit autour d'une démarche intra-simonienne, soit autour d'études comparatives" ${ }^{1}$. Disons que "Lire L'Herbe » d'Alastair Duncan pour l'édition de poche de $1986^{2}$ et Understanding

1 Citons notamment (hors articles de presse)

-Jacques Guicharnaud, « Remembrance of Things Passing : Claude Simon », Yale French Studies, $n^{0}$ 24, Summer 1959, "Midnight Novelists", pp. 101-109.

- Jean-Luc Seylaz, « Du Vent à La Route des Flandres : la conquête d'une forme romanesque », in Un nouveau roman? Recherches et tradition, sous la direction de J. H. Matthews, Le Revue des Lettres modernes, $n^{\circ}$ 94-99, M. J. Minard, Lettres modernes, Paris, 1964, pp. 225-240.

- Brian T. Fitch, « Participe présent et procédés narratifs chez Claude Simon », ibid. pp. 199-216.

- Gérard Roubichou, Lecture de L'Herbe, op. cit.

- Joan C. Stevens, "L'évolution des images entre L'Herbe et Les Corps conducteurs», Claude Simon, Colloque de Cerisy dirigéparJean Ricardou, «10/18», UGE, 1975, pp. 375-382.

- "L'Herbe », in J.A.E. Loubère, The Novels of Claude Simon, Cornell University Press, Ithaca and London, 1975, pp. 74-85.

- «The Grass», in Salvador Jiménez-Fajardo, Claude Simon, Twayne Publishers, a Division of

G. K. Hall Co, Boston, 1975, pp 35-53.

- "The Grass and The Middle Age of Mrs. Eliot », in John Fletcher, Claude Simon and Fiction Now, Calder and Boyars, London, 1975, pp. 135-155.

- Ghislain Bourque, «La Parabole. Analyse végétale de L'Herbe », Etudes littéraires, numéro consacré à Claude Simon, Presses de l'Université Laval, vol. 9, no 1, avril 1976, pp.161-187.

- Diane R. Leonard, «Simon's L'Herbe: Beyond Sound and Fury », The French American Review, vol. 1, no 1, Winter 1976, Texas Christian University, Fort Worth Texas, pp. 13-30.

- «L'Herbe : débuts de la composition symétrique, le livre sur rien » in Stuart Sykes, Les romans de Claude Simon, coll. «Arguments », Les Éditions de Minuit, Paris, 1979, pp. 42-60.

- Doris Y. Kadish, Practices of the New Novel in Claude Simon's L'Herbe and La Route des Flandres, York Press, Fredericton, Canada, 1979,107 pages.

- Karen Gould, "The Faces of Language in The Grass» in Orion Winded, Essays on Claude Simon, edited by Randi Birn and Karen Gould, Lewisbourg: Bucknell University Press, London and Toronto, Associated University Presses, 1981, pp. 72-86.

- Alastair B. Duncan, "Claude Simon : La crise de la représentation » in Critique, «La terre et la guerre dans l'œuvre de Claude Simon », Les Éditions de Minuit, Paris, novembre 1981, pp. 1181-1200, notamment 1186-1188.

2 Alastair Duncan, "Lire L'Herbe », postface à l'édition de poche de L'Herbe, Paris, Les Éditions de Minuit, « Double », Paris, 1986, pp. 185-202. 
Claude Simon de Ralph Sarkonak en 1989-1990 en constitueraient les dernières manifestations.

Comme cela a souvent été dit, s'y affirme une prépondérance anglo-saxonne : car c'est en Grande-Bretagne et en Amérique du Nord que l'œuvre de Claude Simon a été d'abord reconnue comme objet d'enseignement et de recherche universitaire et que L'Herbe a fait l'objet des études les plus spécifiques ${ }^{2}$. Dans beaucoup de ces travaux, le souci majeur semble avoir été de «faciliter » l'accès (des étudiants avancés - graduates -plus que du public en général) à la lecture d'un auteur français " émergent», en examinant, chez certains, ses « attaches » avec la littérature anglo-saxonne (Faulkner ${ }^{3}$, Conrad, Angus Wilson), en soulignant, chez d'autres, la " nouveauté » de son écriture et, le cas échéant, en démontrant combien, voire comment il se distinguait de ses contemporains, en premier lieu, les écrivains dits du Nouveau Roman, puisque, pendant un certain temps, cette étiquette a collé à Claude Simon et que nombre d'études en Amérique du nord ont pris cette appartenance présumée comme point de départ ou comme repoussoir 4 .

Les auteurs de ces travaux anglo-saxons dont plusieurs étaient en français étaient guidés par un souci " pédagogique »d'initiation à une œuvre qui pouvait paraître déroutante dans le cadre des études de littérature française : ils cherchaient à faire apparaître certains caractères qui étaient à prendre en compte dans l'évolution de l'œu-

Ralph Sarkonak, Understanding Claude Simon, University of South Carolina Press, 1990.

2 On constate que la traduction en anglais précède toujours toutes les autres traductions (Voir Entretiens, op. cit. « Editions en langues étrangères »).

3 Non sans humour, Henri Peyre remarquait que le titre du roman de William Faulkner (en français), Tandis quej'agonise aurait « été plus adapté et moins énigmatique » (French Novelist of Today, Oxford University Press, 1967, p. 377).

4 Pour citer un exemple personnel, j'ai moi-même été invité en 1966-67 par l'Université de Californie à Berkeley à donner un cours aux étudiants avancés sur le Nouveau Roman à partir des «Trois romans de 1957: Le Vent, La Modification, La Jalousie. » 
vre de Claude Simon ${ }^{1}$, à saisir les points de cohérence de cette dernière et, partant, en faciliter la compréhension, mais au risque d'en détourner le grand public! Rien d'étonnant à ce que, lorsque les Éditions de Minuit publient en 1979 Les romans de Claude Simon, elles aient fait appel à un Écossais, Stuart Sykes ${ }^{2}$, qui applique strictement pareille approche à l'intention d'un public français qu'il s'agit de rassurer tout en le préparant à la «nouveauté » du romancier.

Dans la quasi majorité de ces travaux, L'Herbe occupe une place bien définie, celle d'être la première étape dans le développement de l'œuvre conçue comme « in Progress $»^{3}$. Au point que ce roman apparaît principalement comme une production de "transition», menant du Vent à La Route des Flandres, se distinguant du premier par une « rupture» (il est à l'origine d'un «tournant» après " une lente évolution par tâtonnement $»^{4}$ ) et du second qui manifestement l'amplifie au point de le rejeter dans l'ombre ou, tout au moins, dans la pénombre. C'est encore ce que l'on trouve, en 1994, comme élément caractéristique de L'Herbe, dans Adventures in Words d'Alastair

${ }^{1}$ Je renvoie à la mise en perspective judicieuse que fait, à ce sujet, Raymond GayCrozier dans un compte-rendu du livre d'Alastair Duncan, Adventures in Words, in Claude Simon 3, Lectures de Histoire, textes réunis par Ralph Sarkonak, Lettres modernes Minard, Paris-Caen, 2000, pp. 223-227. Voir aussi Simon, Euvres, Gallimard, "Bibliothèque de la Pléiade », 2006, «Introduction » d'Alastair Duncan, p. XV.

Stuart Sykes, Les romans de Claude Simon, Éditions de Minuit, «Arguments», 1979, 195 pages. Le chapitre consacré à L'Herbe intitulé «1958. L'Herbe : débuts de la composition symétrique, le livre sur rien " se trouve pp. 42-60. La quatrième de couverture du livre en explicite le projet : «La métaphore de YOrion avengle de Poussin, exploration tâtonnante de l'espace dans l'expérience de la durée, ce voyage gigantesque pourrait bien marquer l'ensemble d'une œuvre qui poursuit depuis trente ans sa démarche au sein d'un univers toujours à découvrir, toujours à inventer $»$.

3 Dans une lettre qu'il m'adressait en janvier 1971, Claude Simon déclarait : « Depuis L'Herbe tous mes bouquins ne forment en réalité qu'un seul texte dont chacun constitue une étape. Toutes proportions gardées, bien sûr, entre Joyce-legéant et moi, je crois que si l'on voulait donner un titre à l'ensemble, le meilleur serait en effet le fameux et toujours provisoire Work in Progress... ».

4 « Du Tricheur au Venty compris [...], il n'y a pas à proprement parler de coupure nette, mais plutôt une lente évolution par tâtonnement. Avec L'Herbe, par contre, il me semble que quelque chose d'assez différent s'est produit. Un tournant était pris..." ("Réponses de Claude Simon à quelques questions écrites de Ludovic Janvier», Entretiens, 1972). Mais Claude Simon ne donne aucune précision sur la nature de ce « tournant». 
Duncan, qui semble confirmer de façon claire et nette ce statut « transitionnel»: du reste, L'Herbe y est désormais traité en moins de trois pages dans un long chapitre consacré à La Roule des Flandres auquel elle sert de «tremplin $»^{1}$.

À ce point de ce bref panorama, on se doit de mentionner les deux Colloques de Cerisy, celui de 1971, Nouveau Roman : hier, aujourd'hui, où Claude Simon n'occupait pas plus de place que les « comparses » qu'on lui avait donnés dans le « Nouveau Roman», et celui de 1974 qui lui est, en revanche, entièrement consacré. Même s'ils affirment la présence incontournable, dans les études « romanesques » d'alors, de Jean Ricardou et manifestent, surtout dans celui de 1974, sa domination idéologique (voir sa communication « Claude Simon, textuellement »), ils traduisent bien de l'un à l'autre la progression de Claude Simon dans le monde universitaire français et en français. Ainsi, notamment dans le Colloque de 1974, les critiques étrangers y sont quasiment réduits à la portion congrue ${ }^{2}$.

Et justement, pour ce qui constitue l'objet de ma présentation aujourd'hui, ces colloques évacuent toute analyse importante de L'Herbe. Seule la brève communication de Joan Stevens dans le colloque de 1974 sur « L'évolution des images entre L'Herbe et Les Corps conducteurs » prend en compte ce roman, mais elle apparaît, avec le recul, tellement limitée par le temps imparti à son auteur dans une séance un peu fourre-tout qu'elle ne peut constituer une étape critique significative.

La seconde grande orientation des études simoniennes est postérieure au Prix Nobel. À l'exception de Writing the Visible de Celia Britton (1987) publié en anglais ${ }^{3}$, elle témoigne de l'émergence d'une

1 Alastair Duncan, Adventures in Words, op. cit., notamment pp. 11-29. "Like Le Vent, and more explicidy than L'Herbe, La Route des Flandres is an attempt to reconstruct the past and hence a search for knowledge » (p. 17) et «Like L'Herbe but more so, La Route des Flandres... » (p. 23).

2 Nouveau roman : hier, aujourd'hui. 1. Problèmes généraux 2. Pratiques, sous la direction de Jean Ricardou et de Françoise Van Rossum, UGE, 1972, respectivement 444 et 440 pages. Claude Simon, Colloque de Cerisy dirigé par Jean Ricardou, UGE, 1975, 447 p. Réédité en 1986 par les Impressions nouvelles sous le titre Lire Claude Simon. Colloque de Cerisy, $470 \mathrm{p}$.

3 Celia Britton, Writing the Visible, Cambridge University Press, Cambridge, Great Britain, 1987, 232 p. 
critique de haut niveau de langue française avec successivement Claude Simon de Lucien Dällenbach (1988) ${ }^{1}$, Les Carrefours du texte de Ralph Sarkonak (1990), Lire Claude Simon. La polyphonie du monde de Patrick Longuet $(1995)^{2}$ et, plus récemment, Ce Grand Temps. Tissai sur l'œuvre de Claude Simon de Mireille Calle-Gruber (2004) ${ }^{3}$. Bien que fondamentalement différents dans leur projet, leur méthodologie et leur écriture, tous ces ouvrages ont en commun de chercher à rendre compte de l'œuvre simonienne dans son ensemble non plus à travers la succession, l'emboîtement de ses textes individuels, mais à partir de points d'entrée multiples et complexes que je schématise modes de représentation, langage mimétique, langage producteur, références, textualité/intertextualité, interférences, réseaux polyphoniques, "révolution d'écriture ", marqueterie du texte ou "inlassable réancrage du vécu ", "arborescence textuelle», etc. Ils incluent, en outre, les œuvres «non romanesques» et enfin s'appuient sur une méthodologie critique et un langage plus contemporains issus notamment des travaux de Roland Barthes, Jacques Derrida, Julia Kristeva, Michel Serres, Tzvetan Todorov, etc., mais aussi des productions simoniennes les plus récentes ${ }^{4}$.

Quand L'Herbe est cité dans ces ouvrages, ce qui n'est pas le cas pour au moins deux d'entre eux, ce roman n'y occupe plus vraiment une place significative. Il est, à l'occasion, convoqué en citations brèves pour venir en appui à la thèse du critique (par exemple l'intertextualité chez Britton) ou pour fournir un exemple supplémentaire dans le cadre d'une démonstration. Le roman est devenu, au mieux,

1 Lucien Dällenbach, Claude Simon, collection « Les Contemporains », Le Seuil, 1988, 221 p.

2 Patrick Longuet, hire Claude Simon. La polyphonie du monde, Éditions de Minuit, «Critique », 1995,157 p.

3 Mireille Calle-Gruber, Le Grand Temps. Essai sur l'œuvre de Claude Simon, Presses Universitaires du Septentrion, "Perspectives », 2004, 260 p.

4 Ralph Sarkonak, Les Carrefours du texte, p. 10 et p. 15, avec notamment cette étonnante déclaration : «De nos jours, l'emploi de la théorie littéraire n'a plus besoin d'être justifié. Il suffit et il faut que l'on dise qu'une large partie des textes de Simon resterait illisible au lecteur qui ne disposerait pas de certaines nouvelles méthodes de lecture inspirées par la poétique moderne. Du reste, il est fort possible que les textes de Simon n'aient pas été tout à fait étrangers à l'élaboration de ces nouvelles méthodes dans la mesure où ils mettent en cause certains critères traditionnels comme le caractère, voire l'unicité du personnage. » 
une des composantes implicites du "Texte simonien», selon les termes de Ralph Sarkonak, mais sans qu'on cherche à l'inscrire dans la progression qui le constitue.

On peut avancer que l'œuvre de Claude Simon désormais consacrée n'a plus, semble-t-il, besoin de cet effort « pédagogique » qu'on observait auparavant. C'est, sans doute, qu'en se développant, à partir des Georgiques (1981) notamment, dans des directions nouvelles, elle exige d'autres lectures qui s'insèrent également dans la production de nouveaux textes simoniens qui, après la césure 1975-1981, vont se succéder. C'est du reste sur eux que va bien vite se focaliser l'attention critique au point de susciter un ensemble impressionnant de travaux et de monographies qui s'éloignent, La Route des Flandres excepté (exception qui mériterait une réflexion), des premières productions.

Un certain « retour» sur L'Herbe s'effectue à travers d'autres travaux concrétisant ce que j'ai appelé une troisième approche, l'approche thématique transversale : je pense principalement au livre de Bernard Andrès en 1992 sur « les profils du personnage » et les " avatars de tous les relais énonciateurs et focalisateurs ", qui consacre de nombreuses pages à cette problématique dans L'Herbe, à celui de Peter Janssens en 1998 sur le travail d'écriture de l'histoire et enfin à celui de Claire de Ribaupierre en 2002 sur le « roman généalogique $»^{1}$. Ce dernier ouvrage démontre que «la chronique de la vie familiale » n'exclut pas un travail de composition et d'écriture ; il étudie toutes les interrogations que suscitent les dispositifs généalogiques en rapport avec la narration et de la référence au "vécu » à partir de L'Herbe et dans toute l'oeuvre simonienne d'une façon extrêmement stimulante.

1 Bernard Andrès, Profils du personnage chez Claude Simon, Editions de Minuit, « Critique », 1992, 284 p ; Peter Janssens, Claude Simon. Paire l'histoire, Presses Universitaires du Septentrion, 1998, 200 p ; Claire de Ribaupierre, Le roman généalogique - Claude Simon et Georges Perec, Éditions La Part de l'œil, 2002, 366 p. 


\section{L'Herbe et le «roman » simonien}

Une lecture de L'Herbe en 2006 pourrait évidemment se passer de tout le poids des études critiques qui s'y sont attachées. J'en suis bien conscient. Et, du reste, la communication d'Anne-Yvonne Julien en sera notamment une démonstration exemplaire. Mais le statut qui s'en dégage ne peut laisser indifférent surtout dans le cadre d'une réflexion comme celle d'aujourd'hui. Pour ma part, je reconnais qu'à trente ans de distance, la lecture d'un même roman ne peut plus se faire dans la même perspective. Le lecteur que j'ai pu être alors n'est, d'abord, plus le même et, en l'occurrence, l'enrichissement progressif de l'ensemble de l'œuvre de Claude Simon - le sien propre dû à son développement et celui apporté par ces différentes "lectures" - est tel qu'il ne peut pas ne pas influer sur notre propre lecture. Le regard que nous portons désormais sur les œuvres " anciennes » a singulièrement changé, d'autant que la disparition de l'écrivain laisse un corpus définitif et donc une totalité vers laquelle on peut se retourner. Peut-être est-ce aussi ce qui nous aidera à comprendre la fortune ou l'infortune de L'Herbe et quelques-unes des raisons qui y ont contribué. Mais tout cela ne peut être qu'esquissé.

Ce qui ressort de tout ce panorama auquel je me suis référé même si j'imagine que tous les simoniens qui nous entourent en connaissent les principales données, c'est qu'une attention distante a été donnée à ce qui pourrait caractériser la nature de ce terme, "roman », qui, comme je l'ai déjà indiqué, désigne L'Herbe et toutes les œuvres de « fiction» simoniennes qui l'ont suivi. Pareille tentative dépasse bien évidemment le cadre d'un exposé et surtout celui de sa conclusion et devrait faire l'objet de travaux plus complexes qui permettraient sans doute non de classifier les œuvres, mais de percevoir l'ampleur de la nouveauté et de la modernité de Claude Simon dans ce genre « inconcevable» (comme disait Valéry) qui n'a cessé, tout au long de son histoire, de se caractériser par sa plasticité permanente et auquel Claude Simon a apporté une contribution exceptionnelle.

In fine, je proposerai, pour susciter la réflexion et le débat, une description provisoire du «roman» simonien en le caractérisant comme « une production textuelle» (précisément « travail sur l'écriture ") à base de vécu fonctionnant comme une fiction à partir de propriétés narratives, compositionnelles, scripturales et thématiques 
spécifiques $»^{1}$. Et je tenterai de mesurer brièvement ma lecture de $L^{\prime}$ Herbe à l'aune de ces considérations.

Production textuelle, L'Herbe l'est dans la mesure où s'y observe un travail d'écriture qui n'a plus rien à voir avec les canons de la narration traditionnelle en opposition à la fiction: le statut singulier de l'instance narrative en reste la principale manifestation marquant, comme le souligne Andrès, les débuts de "l'effacement du personnage » et au-delà un bouleversement du rapport entre le récit et le discours. Le narrateur « objectif » de L'Herbe est aussi un narrateur qui se manifeste de diverses façons comme un "je», mais sans s'identifier à un "narrateur » et encore moins à l'auteur, tout en révélant à maintes reprises son travail « narratif». Sans parler des « déplacements» constants du point de vue spatial, temporel (« un peu plus tôt») avec leurs conséquences visuelles et auditives qui marquent les descriptions et que le travail de Bérénice Bonhomme sur « l'écriture cinématographique » de Claude Simon ${ }^{2}$ éclaire d'une façon nouvelle, cette « révolution narrative » d'un narrateur qui prépare à celui de La Rouie des Flandres qualifié fort justement par Dominique Viart de narrateur qui « ne change pas (à la réserve de l'alternance pronominale $»^{3}$ ) ira en s'amplifiant avec les romans suivants. Elle fait de L'Herbe un ensemble animé d'un véritable mouvement «brownien» entre les modes temporels (passé, présent, participe), les lieux, les objets, puisque le trajet narratif passe indifféremment de descriptions de scènes, d'objets, de personnages à des descriptions de photographies, des transcriptions de cahiers, de listes, etc. dans un incessant va-et-vient de prolifération qui se construit en s'interrompant, puis en se reprenant à l'aide d'une phrase qui s'y

J'emprunte ces trois derniers qualificatifs à Lucien Dällenbach (« Le tissu de mémoire », Postface à l'édition de poche de La Rouie des Flandres, p. 299). Voir aussi cette remarque d'Alastair Duncan : "Au fil des années et des livres, ce sera de plus en plus directement sa propre vie qui fournira à Simon la matière de ses romans. Romans "à base de vécu», dira-t-il, mais indubitablement romans, car la matière est toujours distanciée par la forme, subordonnée aux exigences de la forme et, par les multiples résonances qui ainsi se créent, d'autant plus émouvante » $(C E, \mathrm{XI}-\mathrm{XII})$. La remarque « indubitablement romans » laisse ouvert tout le champ à l'analyse de la nature spécifique du "roman » simonien.

2 Bérénice Bonhomme, Claude Simon. L'écriture cinématographique, L'Harmattan, $2005,156 \mathrm{p}$.

3 Dominique Viart, Une mémoire inquiéte. La Route des Flandres de Claude Simon, PUF, Paris, 1997, p.70. 
révèle déjà comme un des fondamentaux de l'écriture simonienne, au point qu'on en retrouverait certaines «traces » dans Le Tramway qu'un demi siècle sépare pourtant de L'Herbe ${ }^{1}$.

La composition du roman elle-même mérite un instant d'attention. Monolithique, sans chapitre ni section, L'Herbe est un texte dont la composition en spirale (autour des trois scènes entre Louise et son amant) s'inscrit dans un ensemble délimité par un "Rien » initial à quoi répond un « rien » final. En quelque sorte un livre d'un foisonnement exceptionnel... à partir de « rien »! Mais ce « bloc » est parcouru par un mouvement dialectique, celui du continu et du discontinu qui est propre à toute la production simonienne. Avec, toutefois, ici, une particularité qui définit le projet spécifique de L'Herbe, c'est que l'œuvre reste tributaire d'une dimension fictionnelle qui a finalement été retenue par ceux qui en font le roman de Louise et d'une famille autour de la mort d'un vieille tante. Du reste, il n'est pas sans intérêt de suivre à travers le texte la ligne directrice « romanesque » que constitue le retour périodique de notations au passé simple à partir desquelles le récit rebondit, bifurque et se développe et qui n'est pas sans conséquence sur cette lecture "romanesque » de l'œuvre. Paradoxalement, l'extrait de L'Herbe choisi par le Ministère de l'Éducation nationale en 1985 pour " présenter " Simon aux élèves devient emblématique de ce statut du récit/discours narratif inauguré en 1958, autant et sinon plus que sa dimension sociale pour laquelle il avait été choisi comme objet d'étude par l'administration.

Le roman est fortement inspiré d'un "vécu » simonien, l'histoire d'une tante et de sa famille. Elle nous présente certains des protagonistes des romans suivants (Georges, Pierre, Sabine, de Reixach), mais seule la lecture des romans qui lui succèdent nous permet, comme l'a remarquablement montré Claire de Ribaupierre, d'en percevoir toutes les variantes qu'ils entraînent et qui font progressivement basculer la référence qui pourrait être perçue comme autobiographique vers une direction plus féconde - celle de Georges

Pour parodier Henri Peyre, déjà cité (p. 85), Le Tramway serait une sorte de Tandis qu'il agonise. Pour ma part, comme je l'ai étudié dans un texte non publié et intitulé "Un tramway nommé décrire ", ce roman retrouve des données fondamentales de l'univers simonien ouvert par Le Vent et L'Herbe, notamment pour ce qui est de la description. 
dont le roman de 1981, Les Géorgiques, peut être interprété, selon les termes de Lucien Dällenbach, comme un « surdéterminé [qui] renvoie aussi à la geste de Georges " ${ }^{1}$. Cette "réorientation» est sans doute un des éléments qui ont contribué à évacuer L'Herbe de l'ensemble : le vécu n'y est qu'une source pour alimenter la fiction et le travail d'écriture. En effet, Louise et les références qu'elle impliquait ne pouvaient "mener nulle part». En revanche, le transfert vers Georges et toutes les ramifications et substitutions qu'il va entraîner vont ouvrir le roman simonien vers une dimension « autobiographique » de plus en plus présente qui s'accompagnera d'un brouillage narratif, d'une contamination de la voix narrative qui raconte, mais dont le récit est de moins en moins «fiable», sans être pour autant moins prégnant.

Enfin, L'Herbe exprime déjà de façon évidente les axes de la thématique simonienne, la déchéance et la mort à travers le pourrissement ainsi que la dialectique entre Histoire et histoire (voir la citation de Pasternak), ce qui l'inscrit incontestablement dans une dialectique proprement simonienne - celle de l'historicisation de la fiction et de la «fictionnalisation» de l'Histoire. Pour finir, sans vraiment conclure, je dirais que L'Herbe pourrait être caractérisée, au terme d'une production romanesque d'un demi-siècle, comme une sorte d'oeuvre à fonction « séminale». 